\title{
Directing nucleation and growth kinetics in solution-processed hybrid perovskite thin-films
}

\author{
Alexander R. Pascoe ${ }^{1}$, Qinying Gu${ }^{1}$, Mathias U. Rothmann ${ }^{1}$, Wei Li ${ }^{1}$, Yupeng Zhang ${ }^{1}$, \\ Andrew D. Scully ${ }^{2}$, Xiongfeng Lin ${ }^{1}$, Leone Spiccia ${ }^{3}$, Udo Bach ${ }^{1,2}$ and Yi-Bing Cheng ${ }^{1,4^{*}}$
}

\begin{abstract}
A heightened understanding of nucleation and growth mechanisms is paramount if effective solution processing of organic-inorganic perovskite thin-films for optoelectronic applications is to be achieved. Many fabrication techniques have been utilized previously to develop high-performance perovskite layers but there remains an absence of a unifying model that describes accurately the formation of these materials from solution. The present study provides a thorough analysis of nucleation and growth kinetics underpinning the development of hybrid organic-inorganic perovskite thin-films. Through precise control of the perovskite growth conditions the spacing of heterogeneous nucleation sites was varied successfully from several hundred nanometers to several hundred microns. The crystalline regions surrounding these nuclei were found to comprise clusters of highly-oriented crystal domains exceeding $100 \mu \mathrm{m}$ in diameter. However, no beneficial correlation was found between the size of these well-oriented grain-clusters and the optoelectronic performance. The formation of the perovskite microstructure features characteristics of both classical and non-classical growth mechanisms. The insights into perovskite thin-film growth developed by the present study provide clear implications for the development of future hybrid perovskite microstructures.
\end{abstract}

Keywords: organic-inorganic perovskite, nucleation and growth, solar cells

\section{INTRODUCTION}

Since first reported [1], solution-processed hybrid organicinorganic perovskites have garnered significant attention in a range of burgeoning technological fields. Initial studies into organic-inorganic perovskites centered on their application in thin-film transistors and generally explored the synthesis and characterization of highly-ordered, two-dimensional perovskite materials [2]. The real expansion of the research area, however, was driven by studies into perovskite-based photovoltaics and their potential optoelectronic applications. In just seven years, the champion efficiencies for perovskite-based solar cells have risen from approximately $4 \%$ [3], to values exceeding $20 \%$ [4]. This rapid ascension in performance is unprecedented in the field of photovoltaics and places perovskite solar cell efficiencies on par with the commercial multi-crystalline silicon and CdTe technologies. Furthermore, hybrid organic-inorganic perovskites have also been promoted as promising materials for light-emitting diodes (LEDs) and memory applications [5-7]. There are noteworthy shortcomings associated with such hybrid organic-inorganic perovskite materials $[8,9]$, but their optoelectronic performance alone is sufficient to suggest a bright future for perovskite-based applications.

One of the frequently touted benefits of hybrid organicinorganic perovskites is their amenability to solution processing [10]. This attribute may potentially permit reductions in both fabrication costs and energy consumption during manufacturing processes, as well as allowing for a higher throughput. Several studies have duly focused on the processability of perovskite-based materials, researching their deposition through a variety of printing media [11]. However, it is important to note that the deposition of the perovskite precursor solution is inextricably linked

\footnotetext{
${ }^{1}$ Department of Materials Science and Engineering, Monash University, Melbourne VIC 3800, Australia

${ }^{2}$ Commonwealth Scientific and Industrial Research Organization (CSIRO), Research Way, Clayton VIC 3168, Australia

${ }^{3}$ School of Chemistry, Monash University, Melbourne VIC 3800, Australia

${ }^{4}$ State Key Laboratory of Advanced Technology for Materials Synthesis and Processing, Wuhan University of Technology, Wuhan 430070, China

*Corresponding author (email: yibing.cheng@monash.edu)
} 
to the resulting material morphology. Many of the rapid advancements in the area of perovskite-based photovoltaics are essentially due to the ability to control the material morphology and on the application of processing techniques that are capable of producing desirable thin-film perovskite structures with optimized properties. Successful deposition techniques have included two-step [12,13], solvent engineering [14,15], gas-assisted [16], solvent-solvent extraction [17], hot-casting method [18], and vacuum flash-assisted methods [19], to name a few. Many of these fabrication methods result in similar microstructures, although there are subtle differences in the nucleation and growth mechanisms in each case. In this regard, a thorough understanding of nucleation and growth kinetics is imperative to the formation of high-performing perovskite thin-films.

Modifications to the heterogeneous nucleation rate and crystal growth dynamics have produced an interesting range of perovskite thin-film microstructures. The two most widely adopted microstructures are the planar and mesoscopic morphologies. Non-conventional textured perovskite structures have been demonstrated by both one-step [20] and two-step [21] methods, and have been shown to exhibit enhanced light absorption and charge-extraction properties. Yet, one of the most important connections between the perovskite microstructure and the optoelectronic performance is arguably related to the perovskite grain size and charge transport/recombination kinetics. Several studies have revealed clear correlations between the perovskite microstructure, charge-carrier mobilities and charge-carrier lifetimes, implying that large perovskite crystals with a low density of grain boundaries are advantageous [22-25]. These findings are in keeping with the relatively high mobilities and low trap-state densities observed in single-crystal organic-inorganic perovskites [26]. In accordance with these discoveries, numerous studies have looked to increase the size of crystalline grains within the perovskite layer in order to bolster the optoelectronic functionality of the material.

The present study explores the nucleation and crystal growth dynamics that define the formation of hybrid organic-inorganic perovskite thin-films. By controlling the supersaturation rate of perovskite solutions, we are able to accurately control the average density of nucleation sites, as well as the size of crystalline regions and the ensuing crystal morphology. Characterizations of both the perovskite material and its function in a photovoltaic assembly reveal that the perovskite material can be grown into large $(100 \mu \mathrm{m})$ polycrystalline grain-clusters. Building on these experimental insights, we identified elements of both clas- sical LaMer dynamics and non-classical oriented-attachment mechanisms that accounted for the formation of the solution-processed perovskite layer.

\section{EXPERIMENTAL SECTION}

\section{Materials synthesis}

Unless otherwise specified, all materials were purchased from either Alfa Aesar or Sigma-Aldrich, and used as received. Spiro-OMeTAD $\left(2,2^{\prime}, 7,7^{\prime}\right.$-tetrakis $(N, N$-di- $p$ meth-oxyphenylamine)-9,9-spirobifluorene) was purchased from Luminescence Technology Corp. $\mathrm{CH}_{3} \mathrm{NH}_{3} \mathrm{I}$ was prepared using a previously reported method [16]. The $18 \mathrm{~nm} \mathrm{TiO}_{2}$ nanoparticle paste (CCIC) used in the solar cell devices was diluted in ethanol (1:8 vol.) prior to spin-coating.

\section{Perovskite thin-film fabrication}

Perovskite thin-films were formed on $\mathrm{TiO}_{2}$-coated fluorine-doped tin oxide (FTO)-glass substrates. The FTO-glass substrates were cleaned using a three-stage sonication process in hellmanex, water and ethanol. The dense $\mathrm{TiO}_{2}$ layer was formed by spray pyrolysis of a bis(isopropoxide)-bis(acetylacetonate)titanium(IV) solution at $500^{\circ} \mathrm{C}$ using an automated ultra-sonic spray nozzle (SonoTec). $\mathrm{TiO}_{2}$-coated FTO-glass substrates were cut into rectangles of approximately $20 \mathrm{~mm} \times 12.5 \mathrm{~mm}$. The films were treated using a UV-ozone system for $10 \mathrm{~min}$ prior to film deposition. The perovskite precursor solution was formed by mixing stoichiometric amounts of $\mathrm{CH}_{3} \mathrm{NH}_{3} \mathrm{I}$ and $\mathrm{PbI}_{2}$ in a combination of dimethyl sulfoxide (DMSO) and $N$-methyl-2-pyrrolidone (NMP) $(7: 3 v / v)$ to form a (46 wt.\%) concentration solution. $30 \mu \mathrm{L}$ of the perovskite precursor solution was applied to the surface of the substrate prior to spin-coating. The films were spin-coated using a two-stage process: $1000 \mathrm{rpm}$ for $5 \mathrm{~s}$ using an acceleration of $200 \mathrm{rpm} \mathrm{s}^{-1}$, then $6000 \mathrm{rpm}$ for $50 \mathrm{~s}$ using an acceleration of $6000 \mathrm{rpm} \mathrm{s}^{-1}$. For the XS, S, M and $\mathrm{L}$ films, the gas flow was introduced after approximately $20 \mathrm{~s}$ into the spin-coating step, and sustained for $28 \mathrm{~s}$ in the high-velocity flow case and $20 \mathrm{~s}$ in the low-velocity flow case. All films were annealed at $100^{\circ} \mathrm{C}$ for $10 \mathrm{~min}$, unless otherwise specified. The XS, S and dendrites films were annealed uncovered, within a $\mathrm{N}_{2}$ filled glovebox. The $\mathrm{M}$ and L films were annealed within a glass petri dish, with $1 \mu \mathrm{L}$ of additional DMSO:NMP $(7: 3 v / v)$ deposited within the petri dish for the $\mathrm{L}$ film. The films were removed from the petri dish and placed directly on the hotplate once they had fully blackened. 


\section{Materials and optoelectronic characterization}

Optical micrograph images were captured using a Nikon Eclipse LV100 POL system. Scanning electron microscopy (SEM) profiles were performed using a FEI Magellan 400 FEG microscope. Images were captured using an accelerating potential of $5 \mathrm{kV}$ and a beam current of $25 \mathrm{pA}$. Transmission electron microscopy (TEM) samples were prepared on thin carbon grids and imaged using a JEOL 2100F FEG-TEM with a Gatan Ultrascan camera, operated at a $200 \mathrm{kV}$ accelerating potential. In order to minimize possible electron beam-induced artefacts, we used a low-dose TEM imaging condition with an electron dose rate of $\sim 1 \mathrm{e} \AA^{-2} \mathrm{~s}^{-1}$. Photoluminescence (PL) mapping images were performed using a confocal microscope system (WITec, alpha 300R) with $50 \times$ and $100 \times$ objective lens (NA $=0.9)$ in ambient conditions. The perovskite samples were excited using a $532 \mathrm{~nm}$ laser source $(5 \mu \mathrm{W})$ and sample translation was performed using a piezo-crystal controlled scanning stage. Time-resolved PL measurements on films were performed using an Edinburgh Instruments Ltd. FLSP920 time-correlated single photon counting (TCSPC) spectrometer, incorporating a $466 \mathrm{~nm}$ pulsed diode laser, with a $\sim 100$ ps pulse width and a laser irradiance of $\sim 40 \mu \mathrm{W} \mathrm{cm}{ }^{-2}$, as the excitation source. X-ray diffraction (XRD) perovskite samples were prepared on $\mathrm{TiO}_{2}$-coated FTO-glass substrates, and analyzed using a D2 PHASER (Bruker Corp.). UV-vis spectroscopy was performed using a PerkinElmer Lambda 950 UV/VIS/NIR spectrometer. Experiments were calibrated using a $\mathrm{TiO}_{2}$-coated FTO-glass substrate.

\section{Solar cell fabrication}

$\mathrm{TiO}_{2}$-coated FTO-glass substrates and perovskite thin-films were prepared as stated above. The hole-transporting layer was prepared by depositing a solution formed by dissolving $40 \mathrm{mg}$ of spiro-OMeTAD in $500 \mu \mathrm{L}$ of chlorobenzene, with $7.5 \mu \mathrm{L}$ lithium bis(trifluoromethylsulphonyl) imide in acetonitrile $\left(500 \mathrm{mg} \mathrm{mL}^{-1}\right)$ and 16.88 $\mu \mathrm{L}$ 4-tert-butylpyridine as additives, directly on the perovskite surface. The spiro-OMeTAD solution was spin-coated at $3000 \mathrm{rpm}$ for $30 \mathrm{~s}$ with an acceleration of $3000 \mathrm{rpm} \mathrm{s}^{-1}$. An $80 \mathrm{~nm}$ gold electrode was then evaporated onto the devices.

\section{Solar cell characterization}

Current-voltage characterization was performed using a solar simulator (Oriel) fitted with a filtered $1000 \mathrm{~W}$ xenon lamp to replicate AM1.5, $1 \mathrm{~kW} \mathrm{~m}^{-2}$ conditions. The illumination of the light source was calibrated using a silicon reference cell (Peccell Technologies) with a color filter to minimize the spectral mismatch between the calibration diode and the perovskite solar cell. The $J-V$ response was recorded using a Keithley 2400 source meter. The solar cells were masked with a non-reflective metal aperture of $0.16 \mathrm{~cm}^{2}$ that defined the active area of the device. Incident photon-to-current efficiency (IPCE) measurements were performed using a $150 \mathrm{~W}$ xenon lamp (Oriel) fitted with a monochromator (Cornerstone 260). The illumination spot-size was slightly less than the $0.16 \mathrm{~cm}^{2}$ aperture. IPCE photocurrents were recorded under short-circuit conditions using a Keithley 2400 source meter. The monochromatic photon flux was quantified via a calibrated silicon cell (Peccell Technologies). Intensity-modulated photovoltage spectroscopy (IMVS) measurements were performed using a $435 \mathrm{~nm}$ diode illumination source powered by a PP210 potentiostat. An AC perturbation of $<5 \%$ of the steady-state intensity was used to modulate the diode illumination source. A Zahner Zennium electrochemical workstation (ECW IM6) was used as a frequency response analyzer, and IMVS measurements were performed in the $500 \mathrm{kHz}$ to $10 \mathrm{~Hz}$ frequency range. Real and imaginary photovoltage data were analyzed using $\mathrm{Z}$ view equivalent circuit modelling software (Scribner).

\section{RESULTS AND DISCUSSION}

\section{Controlling nucleation and growth}

Within solution-processed hybrid organic-inorganic perovskites, fundamental control of the perovskite crystal growth is achieved largely by regulating the density of nuclei and the concentration of the precursor solution. Solution-processed organic-inorganic perovskite thin-films are characterized by intrinsically low nucleation rates, and it is imperative to accelerate the rate of heterogeneous nucleation in order to establish a compact material layer [12]. There are subtle differences in how this is achieved for different perovskite materials; however, we postulate that the overriding theory presented herein is applicable to all solution-processed hybrid inorganic-organic perovskite films. In this study, the focus has been on the formation of methylammonium lead tri-iodide $\left(\mathrm{CH}_{3} \mathrm{NH}_{3} \mathrm{PbI}_{3}\right.$, i.e., $\mathrm{MAPbI}_{3}$ ) from solution, but other systems have also been examined as a proof of concept. The basic fabrication steps used to form the various perovskite microstructures analyzed in this study are shown in Fig. 1a. These steps were used to produce five different perovskite microstructures: perovskite dendrites, as shown in Fig. 1b, and uniform 

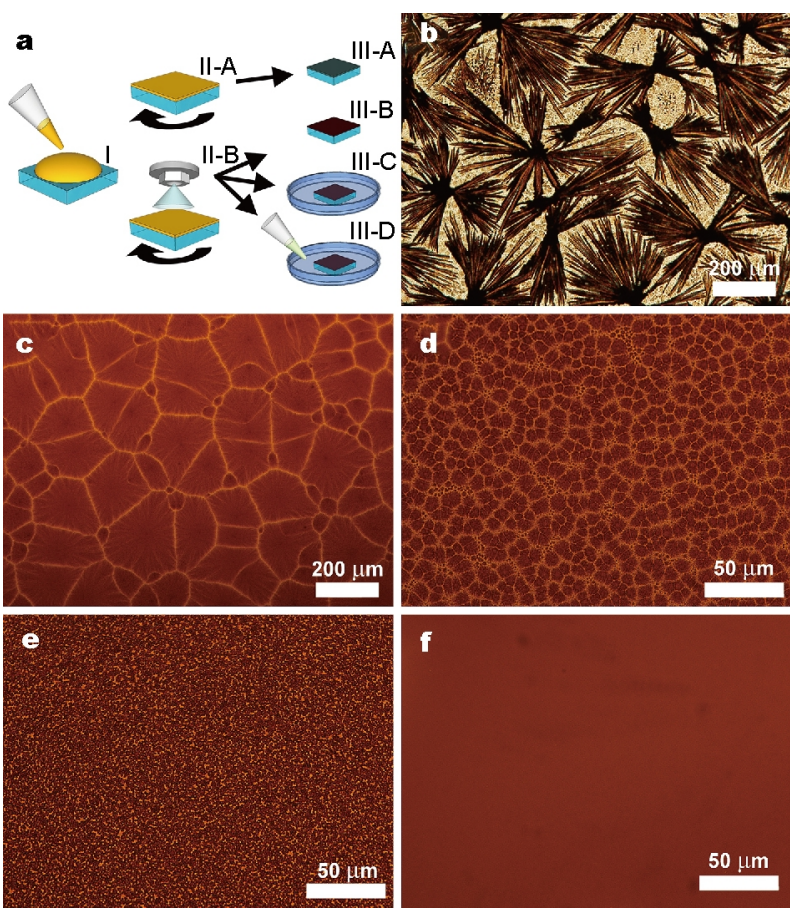

Figure 1 (a) Schematic representation of the perovskite thin-film fabrication process. The process is roughly divided into three stages (left to right): the deposition of the perovskite precursor solution, the spin-coating of the solution, and the annealing of the film. The resulting perovskite microstructures of the dendrites, L, M, S and XS films are shown in the optical microscope images (b-f), respectively. Optical micrographs were taken in transmission mode, and the perovskite films were formed on $\mathrm{TiO}_{2}$-coated FTO-glass substrates.

planar perovskite films comprising large (L), medium (M), small (S) and extra-small (XS) crystalline regions, as shown respectively in Fig. 1c-f. A 46 wt.\% $\mathrm{MAPbI}_{3}$ precursor solution, formed by dissolving stoichiometric amounts of methylammonium iodide (MAI) and lead iodide $\left(\mathrm{PbI}_{2}\right)$ in DMSO:NMP $(7: 3 v / v)$ was used to form the $\mathrm{MAPbI}_{3}$ films. The perovskite solution was initially cast onto a $\mathrm{TiO}_{2}$-coated FTO-glass substrate, and then spin-coated at $6000 \mathrm{rpm}$ to promote solvent evaporation. In the case of the dendrite film, the spin-coating procedure was performed with no additional steps. For the L, M, $\mathrm{S}$ and XS films, a nitrogen gas flow was used to further accelerate evaporation of the DMSO/NMP solvent, as per the gas-assisted fabrication method [16]. The exit aperture of the gas flow was varied to produce a relatively high-velocity flow for the case of the XS film, and a comparatively low-velocity flow for the L, M and S films. Photographs of the different "as-coated" films are shown in Fig. S1. To fully remove the solvent and promote microstructure development, the as-coated films were then annealed at $100^{\circ} \mathrm{C}$. in a nitrogen-filled glovebox for a duration of $10 \mathrm{~min}$ under the following experimental conditions: the dendrites, XS and S films were all annealed on a hot-plate in the nitrogen atmosphere without any cover. The M film was annealed on a hot-plate inside a glass petri-dish until the film turned black, at which stage it was removed from the petri-dish and placed directly on the hot-plate. The L film was placed inside a glass petri-dish along with an additional $1 \mu \mathrm{L}$ of the 7:3 $(v / v)$ DMSO:NMP solvent, which was then placed on the hotplate. Once again, when the film turned black it was removed from the glass petri-dish and placed directly on the hot-plate.

The resulting films (Fig. 1) comprised a range of nucleation densities and material microstructures. In the case of the dendrites (Fig. 1b), the nucleation density is low and the nucleation sites are approximately $100 \mu \mathrm{m}-1 \mathrm{~mm}$ apart with the material forming into distinctive needle-like structures. The planar perovskite morphologies, L, M, S and XS films (Fig. 1c-f) show increased nucleation density with the nucleation sites spaced by $<300 \mu \mathrm{m},<10 \mu \mathrm{m},<$ $2 \mu \mathrm{m}$ and $<500 \mathrm{~nm}$, respectively. The diameters of the crystalline areas growing from these nucleation sites are approximately equivalent to the spacing distance between nucleation sites. SEM images of the grain structure within the XS film are presented in Fig. S2. The average grain diameter $(<500 \mathrm{~nm})$ within the XS thin-films is approximately equivalent to those reported previously for films produced via gas-assisted and solvent-engineering fabrication methods $[15,16]$. The largest crystalline regions depicted in Fig. 1 , are in excess of $100 \mu \mathrm{m}$ (Fig. 1c). Although previous studies have reported similar microstructures using a hot-casting technique at $>170^{\circ} \mathrm{C}[18]$, to the best of our knowledge this is the first time that this type of large grain morphology has been produced using a relatively low-temperature $\left(\leq 100^{\circ} \mathrm{C}\right)$ process.

The major driver for the resulting nucleation density and perovskite morphology in each case is linked to supersaturation of the perovskite precursor solution. Nucleation is only expected to occur when the solution reaches supersaturation. As stated previously, the primary aim of the applied gas-flow is to evaporate the solvent and increase the $\mathrm{MAPbI}_{3}$ concentration in solution. This evaporation process is largely governed by the boiling point of the solvent and the velocity of the gas-flow. The subsequent annealing procedure then removes the remainder of the solvent within the film, leaving only the precipitated perovskite solute. Therefore, it is possible to control the resulting perovskite microstructure by regulating the amount of solvent removed during both the 
gas-blowing and annealing steps. In the method depicted in Fig. 1a, the solvent vapor pressure of the atmosphere surrounding the film was modified in order to control the solvent evaporation rate. The evaporation rate is relatively high when the uncovered $100^{\circ} \mathrm{C}$ film is exposed directly to the $\mathrm{N}_{2}$ atmosphere, and this evaporation rate can be reduced through the use of the sealed petri-dish with additional solvent. A comparable method of controlling the solvent evaporation rate is through regulating the temperature of the annealing process. Fig. S3 demonstrates that a similar control of the nucleation dynamics can be achieved through adjusting the hot-plate temperature between $70^{\circ} \mathrm{C}$ and $110^{\circ} \mathrm{C}$. Furthermore, the link between the solvent evaporation rate and the nucleation kinetics is applicable to other solution-processed perovskite compositions. The optical micrograph images in Figs S4 and S5 illustrate that the nucleation density in solution-processed methylammonium lead bromide and mixed anion/cation perovskite films can also be controlled by adjusting the solvent evaporation rate. These observations reveal the broad applicability of controlling the nucleation density by tuning the solution supersaturation rate in a variety of perovskite thin-films.

Previous studies claimed that similar microstructures produced by hot-casting methods comprise single grains that have diameters in excess of $100 \mu \mathrm{m}[18,27]$. An effective way of testing this hypothesis is by comparing the crystallographic composition of an individual grain using TEM. TEM images were taken for a low nucleation density, large crystalline-region film (Fig. 2) to test whether these large crystalline areas comprise a single grain, as has been reported previously. The films were formed on a carbon-coated TEM grid, as shown by the reflectance mode optical microscope image in Fig. 2a. A bright-field image of the $\mathrm{MAPbI}_{3}$ film within one of the large crystallized regions is presented in Fig. 2b. Eight diffraction patterns were acquired from separate regions within this bright-field area in order to map the crystal orientation of each respective region. The corresponding diffraction patters are presented in Fig. $2 c-j$, with the region number shown in the top right-hand corner of each diffraction pattern. The most significant piece of information derived from the diffraction patterns is that the eight regions share a similar crystallographic orientation (110), highlighted by the common spacing of the diffraction spots (Fig. S6), although the TEM image of Fig. 2b shows a clear polycrystalline microstructure. However, within each separate region the crystal unit is either rotated slightly in the substrate plane or tilted relative to the substrate normal.
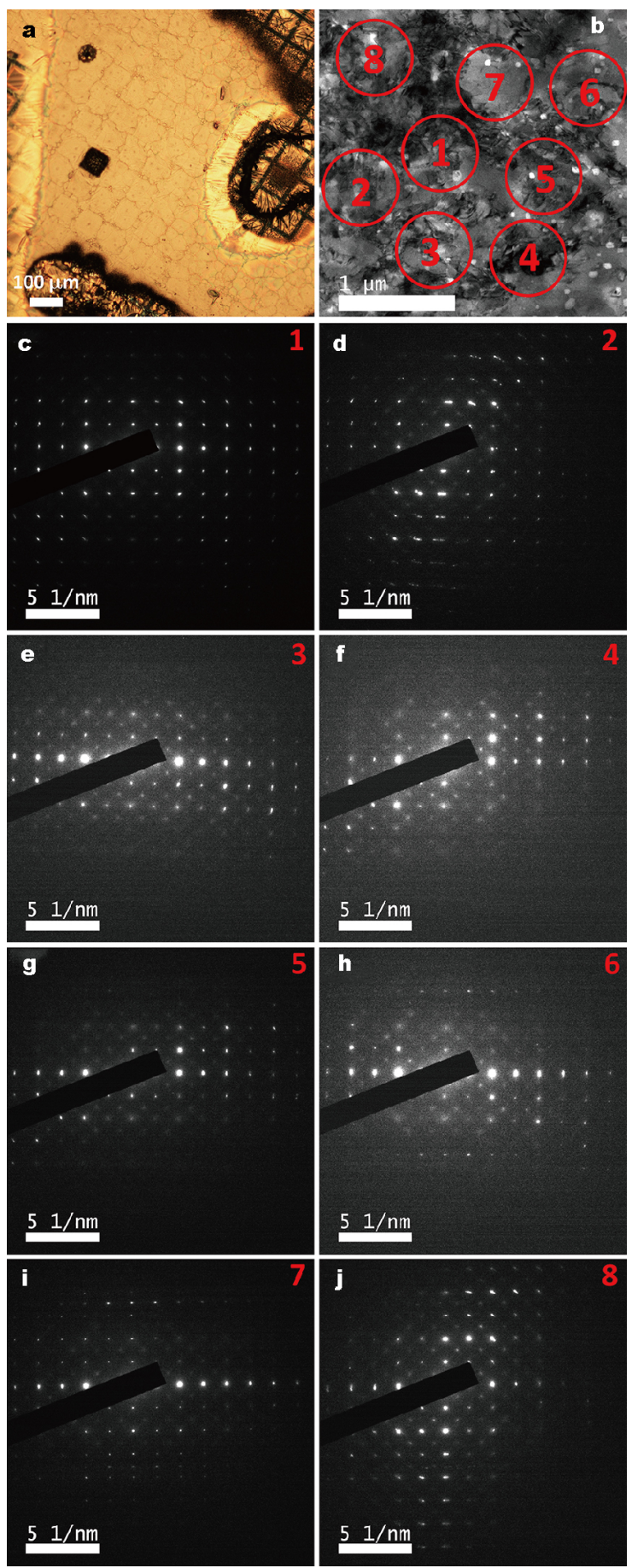

Figure 2 (a) Optical microscope image of the perovskite film, as deposited on the carbon TEM grid. (b) TEM bright field image of the perovskite film shown in (a). The selective area diffraction patterns presented in images $(c-j)$ correspond to the circled areas indexed in the bright field image. Each imaged area is denoted by the number in the top right-hand corner of the diffraction pattern. The TEM diffraction patterns all describe the (110) facet, as shown in Fig. S5 and as identified previously, with the different alignment of the pattern and the differing spot intensities being due to crystal rotation and tilting. 
These slight changes in crystallographic orientation between neighboring crystalline units account for the minor rotation of the diffraction pattern as well as the changing intensity of individual diffraction spots. Furthermore, the splitting and broadening of diffraction spots (e.g., Fig 2d) provide evidence for multiple crystals being present within the one diffraction region.

From these results, the large crystalline areas formed under low nucleation conditions (Fig. 1c) can be concluded to be polycrystalline, and do not comprise a single grain. Given that these large crystalline areas comprise clusters of similarly oriented domains, they are henceforth referred to as perovskite "grain-clusters". This conclusion is further substantiated by SEM images, which depict < $500 \mathrm{~nm}$ grains, as well as revealing clear domain boundaries between crystal regions (Fig. S7). The similar geometries exhibited by neighboring crystal domains (Fig. S7) reflect the similarly oriented nature of the perovskite grains within this relatively large grain-cluster. This is in contrast to the poorly-oriented XS film (Fig. S2), where there is no clear orientation relationship of the surface fringes between neighboring grains. Indeed, there is evidence of domain boundaries within hot-cast films (Fig. S8), implying that similar microstructures produced using alternative methods also comprise multiple $<500 \mathrm{~nm}$ sized grains, and not the $>100 \mu \mathrm{m}$ single crystal grains that have been claimed previously [18]. We suspect that films formed under hot-casting conditions share the similar preferential oriented growth mechanisms that are revealed in the present study from both TEM diffraction patterns (Fig. 2) and TEM dark field images (Fig. S9). Hence, the large grains observed in optical microscope images appear to be merely clusters of similarly-oriented crystal domains rather than uniform single crystals.

\section{Nucleation and growth models}

Nucleation and growth in solutions chiefly determine the particle size and morphology of materials, and these processes are governed by both thermodynamic and kinetic factors. Based on existing experimental insights into the nucleation and growth kinetics of solution-processed hybrid organic-inorganic perovskites, it is possible to propose a nucleation and growth model which formalizes the establishment of these perovskite microstructures. At the heart of this growth model is the initial formation of heterogeneous nucleation sites at the solution/substrate interface. It is important to again stress the intrinsically low heterogeneous nucleation rates characteristic of hybrid organic-inorganic perovskite solutions. In the absence of nucleation control, the relatively high crystal growth rates endemic to this class of perovskite solution inevitably lead to the poor surface coverage of the underlying substrate. In this regard, a careful balance is required between the nucleation and growth rates. The classical LaMer curve [28] (Fig. 3a) describes the nucleation and growth kinetics of solutions, largely as a function of the solution supersaturation, and relates directly to the growth of perovskite thin-films. The curve is typically divided into three regions relating to: (I) the initial increase in concentration of the solution; (II) the
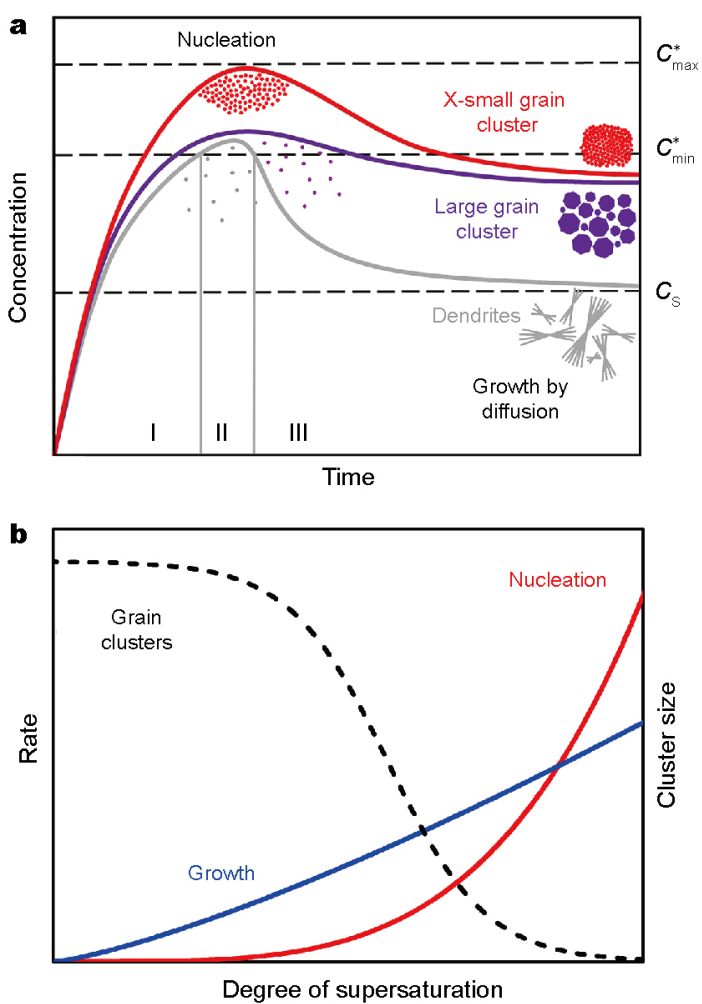

Figure 3 Nucleation and growth models used to describe the relationship between the solution concentration and the nucleation density. (a) The classical LaMer curve [28] divides the crystal growth into 3 distinct phases. These three phases are presented for the XS (red trace), L (purple trace) and dendrites (grey trace) films used in the present study. In the first phase (I), as the solvent evaporates, the concentration curve crosses the solute solubility limit $\left(C_{\mathrm{S}}\right)$ and approaches supersaturation. In the second phase (II), the solution reaches supersaturation, and the ensuing nucleation density is proportional to the area under the curve enclosed between the minimum $\left(C_{\min }^{*}\right)$ and maximum $\left(C_{\max }^{*}\right)$ supersaturation limits. In the third phase (III), the concentration curve moves below the supersaturation minimum, and the precipitation of solute material brings about a further decrease in the solution concentration. (b) The nucleation (red trace) and growth (blue trace) rates as a function of the supersaturation degree, and how this then determines the grain-cluster size (dashed black trace). The polynomial progression of the nucleation and growth rates, as a function of the supersaturation, has been well established in previous studies [46]. 
formation of nuclei as the concentration reaches supersaturation; and (III) the subsequent crystal growth accompanied by a reduction in solution concentration. The competition between nucleation and growth kinetics is a function of the solution supersaturation and largely determines the resulting size of each crystalline region (Fig. 3b). In the present study, the degree of the solution supersaturation broadly dictates the density of nucleation sites forming at the substrate/solution interface. The purpose of the $\mathrm{N}_{2}$ gas flow in the gas-assisted spin-coating method is to accelerate evaporation of the solvent, shortening the time for the solution to reach supersaturation and maintaining the solution in a state of supersaturation for a longer duration (red trace, Fig. 3a). It can also bring the solution close to the point of supersaturation (purple trace, Fig. 3a) followed by an annealing process to push the solution into a state of supersaturation. Both the degree of supersaturation and the duration that the solution is supersaturated define the number and the size of initial nuclei in the solution. Heterogeneous nucleation occurs on the $\mathrm{TiO}_{2}$ surface. These nuclei then grow through solute precipitation until this growth is impinged upon by neighboring grains. Effective control of the solution supersaturation is the primary method by which the resulting perovskite microstructure is able to be controlled. It should be stressed that all perovskite crystal formation happens in the presence of solvent on the substrate, which is gradually removed during drying and annealing. At this point, the crystal grains precipitate as a result of supersaturation in the precursor solution on the substrate, and therefore the conditions for LaMer crystal formation are fulfilled.

To a first approximation, the classical LaMer curve [28] provides a satisfactory description of nucleation and growth kinetics for solution-processed perovskite systems. Time-lapse optical microscope images (Fig. S10) accurately capture the point of nucleation and the ensuing crystal growth, resulting in the large grain-clusters seen in Fig. 1c. However, classical models dictate that growth and solute precipitation will dominate after the initial nucleation stage and no new nucleation sites will form unless the solution again reaches supersaturation. The fact that the large crystalline regions seen in Fig. 1c constitute clusters of well-oriented grains instead of mono-crystalline domains indicates that classical models alone cannot account for the growth of solution-processed hybrid perovskite thin-films. Non-classical theories describing oriented-attachment [29-31] provide a better account of the subsequent growth of the well-oriented, polycrystalline grain-clusters. Under this interpretation, solution-based nanoparticle agglomerates are capable of anchoring to existing perovskite crystals and shifting their crystallographic orientation in order to minimize the surface free energy. Previous studies have reported the surface energy reductions associated with the strong surface adsorption characteristics of oriented-attachment mechanisms $[32,33]$. Oriented-attachment processes appear to be consistent with the well-oriented polycrystalline microstructures observed in this study, although there is currently only limited evidence to suggest that particle agglomerates exist within the solution prior to film formation $[34,35]$. There is also strong evidence to suggest that intermediate phases and Lewis-base adducts play a profound role in the crystallization dynamics of organic-inorganic perovskite-based films [36,37].

Previous analysis of large-grain hybrid organic-inorganic perovskite growth mechanisms has revealed the formation of Volmer-Webber like 'islands' which emerge from a central nucleation site [27]. These islands grow in a radial fashion through adatom-adatom interactions until their growth is impinged upon by a neighboring island, resulting in a distinctive Voronoi polygon microstructure. However, classical atom-mediated growth models are unable to satisfactorily explain the polycrystalline nature of the resulting perovskite microstructure observed in the present work. Polycrystalline microstructures have similarly been observed in hybrid organic-inorganic perovskite dendrites $[16,25]$, despite the comparatively low heterogeneous nucleation rates. Other recent studies have also revealed highly-oriented growth of perovskite domains within dendritic morphologies [38], involving the growth mechanisms associated with perovskite dendrites that appear to be very similar to those outlined above. Comparisons between the growth of planar and dendritic perovskite morphologies are discussed further in the Supporting information (Figs S11 and S12). Given these observations, it is unlikely that perovskite thin-films with single crystalline grains in excess of $10 \mu \mathrm{m}$ can be grown directly from solution, in contrast with the formation of millimeter-size perovskite single crystals in solutions, where grains are free to undergo equiaxed growth [26,39]. Increasing the in-plane dimensions of individual grains in thin films might be best achieved through Ostwald ripening mechanisms [40], although even via ripening mechanisms the resulting grain diameters may still be restricted to approximately only three-times the film thickness [41]. 


\section{Material characterization}

One of the key reported attributes for large, perovskite single crystals is a reduction in the density of parasitic trap states [26]. An effective method for observing the relative influence of trap states on the optoelectronic performance is through analyzing the PL response of a material. Within the pristine perovskite film, at relatively low charge injection levels, free-carriers are typically limited to recombining through either radiative bimolecular or non-radiative trap-mediated channels. The relative PL intensity provides an indication of the proportion of charge-carriers recombining through each respective channel. PL maps of the $\mathrm{XS}, \mathrm{S}, \mathrm{M}$ and $\mathrm{L}$ grain-clusters are shown in Fig. $4 \mathrm{a}-\mathrm{d}$, respectively. Regions of contrasting intensity between neighboring grains and grain-clusters can be clearly seen in the PL maps, as identified previously $[25,42]$. Firstly, it is important to note the differing intensity scales for each image in Fig. 4. The different scales for each PL image reveal that the PL signal is comparatively stronger in the XS film and significantly weaker in the $\mathrm{M}$ and $\mathrm{L}$ grain-clusters. This result is also reflected in the normalized PL maps (Fig. S13). Within the S (Fig. 4b) and M (Fig. 4c) grain-clusters, the highest observed PL intensities occur within the relatively

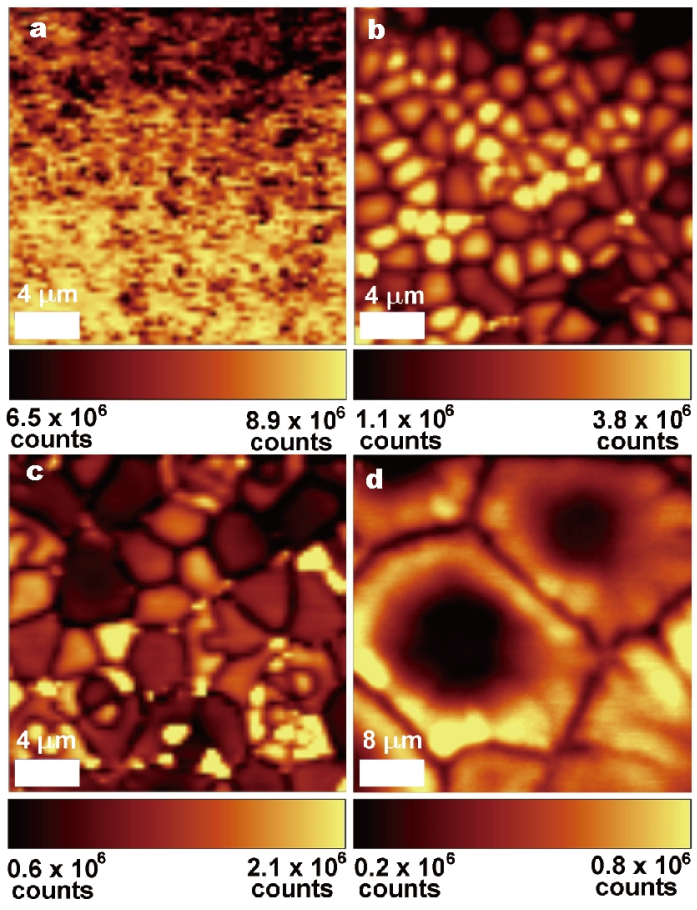

Figure 4 Spatially-resolved PL maps of the (a) XS, (b) S, (c) M and (d) $\mathrm{L} \mathrm{CH}_{3} \mathrm{NH}_{3} \mathrm{PbI}_{3}$ films. The intensity scale for each respective $\mathrm{PL}$ map is shown beneath the image. The PL response was generated using a $532 \mathrm{~nm}$ laser excitation source. Intensity normalized PL maps are presented in Fig. S12. small grain-clusters, while the larger clusters exhibit comparatively lower PL intensities. The relationship between the grain-cluster size and PL intensity mirrors the overall trend in the PL intensity when comparing the L grain-cluster film (Fig. 4d) to the XS film (Fig. 4a). Within the large grain-clusters (film L, Fig. 4d), the PL signal is clearly non-uniform across the surface of the cluster, with the center of the grain-cluster appearing relatively darker than the fringes. This observation is partially due to differences in the thickness of the perovskite layer. However, dark ridges extending from the grain-cluster boundary towards the center of the cluster provide evidence for the existence of defects and crystallographic imperfections. Given the sheer size of the large grain-clusters and the energetically stringent requirements of maintaining highly-oriented crystal growth, it is conceivable that prominent dislocations emerge during the growth process, leading to regions of heightened rates of non-radiative charge trapping. The accelerated creation of point defects, twins, stacking faults, dislocations and misorientation are indicative of materials formed through oriented-attachment mechanisms $[31,43,44]$.

In the PL maps of all four films, the boundaries between grain-clusters display comparatively low PL intensities. Previous reports have also noted the low PL intensities characteristic of grain boundaries [42] and have highlighted the deleterious effects of grain boundaries on the charge-carrier lifetimes [25]. PL decays for each of these morphology types are presented in Fig. 5 and the values of the fitting parameters for each decay are given in Table S1. The PL lifetimes from the four different grain-cluster morphologies show a similar trend to that observed from the PL mapping intensities. The XS film (red trace) exhibited a weighted mean free-carrier lifetime of $4.62 \mu \mathrm{s}$, which is significantly longer than the $0.28 \mu \mathrm{s}$ lifetime measured for the large grain-cluster film (purple trace). The shorter lifetimes and lower relative PL intensities of the large grain-cluster film observed from PL decays and images, respectively, suggest that a higher degree of crystallographic orientation does not necessarily correspond with a higher degree of material quality. It is likely that the 'building-block' assembly synonymous with oriented-attachment growth leads to additional domain boundaries parallel to the underlying substrate, which may also promote non-radiative recombination. The diffraction patterns seen in Fig. 2 and Fig. S9 are very regular and well defined, indicating a relatively high degree of crystal quality within the 'building-blocks' themselves. The relatively lower PL intensity of the large grain-clusters 


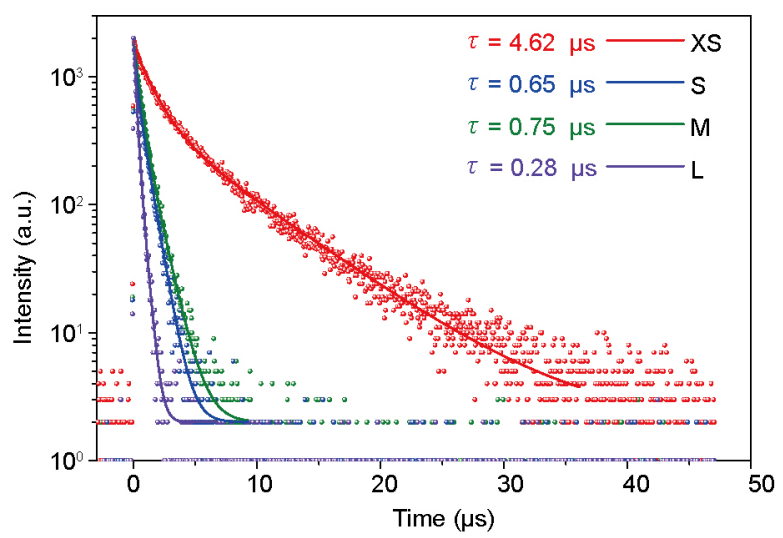

Figure 5 Time-resolved PL decays observed for the XS (red markers), $S$ (blue markers), $M$ (green markers) and L (purple markers) perovskite films. The PL transients were fitted with a double-exponential decay, and the fitting parameters are presented in Table S1.

compared to that of the smaller grains is therefore more likely due to the large number of interfaces between the fine crystalline domains in the large clusters. Furthermore, the PL results imply that the prominent (110) facet, seen from TEM diffraction patterns (Fig. 2 and Fig. S9) and XRD measurements (Fig. S12), may not luminesce as intensely as alternate crystallographic orientations. An investigation into the relationship between the crystallographic orientation and luminescence intensity is outside the scope of the current work, but will be explored in subsequent studies. Nevertheless, the combined spatially-resolved and time-resolved PL measurements support the oriented-attachment interpretation of the perovskite thin-film growth, and do not support predictions obtained from classical growth models.

The performance of perovskite-based solar cells formed from the four material morphologies investigated in the present study reflects many of the observations determined through the materials characterization measurements. Each cell comprised an FTO-glass substrate coated with a dense $\mathrm{TiO}_{2}$ layer, a $\sim 100 \mathrm{~nm}$ thick mesoporous $\mathrm{TiO}_{2}$ layer, the $\sim 350 \mathrm{~nm}$ thick $\mathrm{CH}_{3} \mathrm{NH}_{3} \mathrm{PbI}_{3}$ film, a $\sim 150 \mathrm{~nm}$ spiro-OMeTAD layer and an evaporated $85 \mathrm{~nm}$ thick gold contact. These cells were characterized by current-voltage $(J-V)$ measurements, as shown in Fig. 6. From the $J-V$ characteristics, it is apparent that there is no significant performance difference between the four respective morphologies, with champion devices for each cell type achieving efficiencies of approximately $17 \%$. The comparatively higher open-circuit potentials $\left(V_{\mathrm{OC}}\right)$ observed for the XS solar cells (Fig. 6a) can be accounted
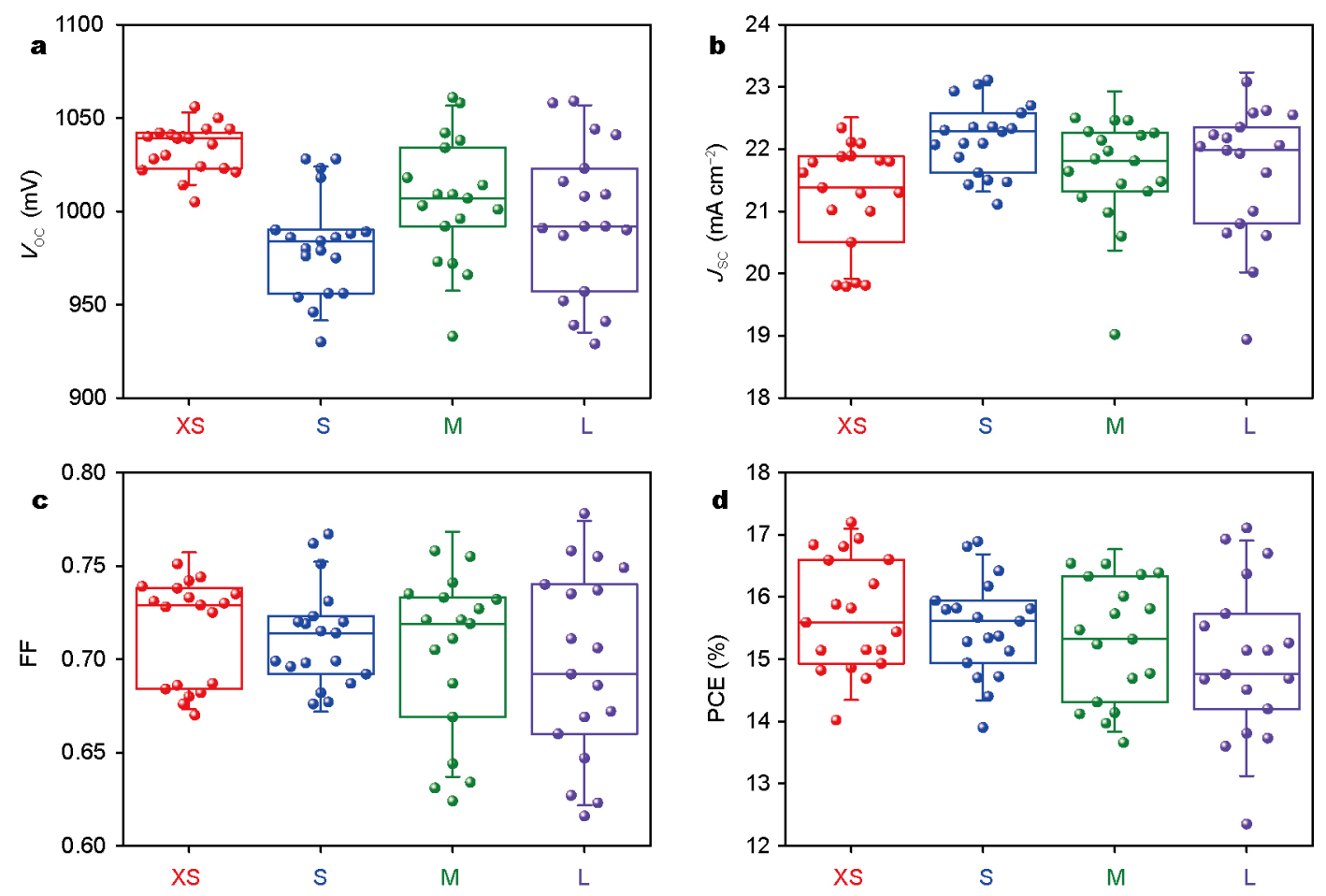

Figure 6 Photovoltaic parameters for the perovskite solar cells formed using the XS (red), S (blue), M (green) and L (purple) film morphologies. 18-19 cells were made for each morphology condition. The open-circuit voltage $\left(V_{\mathrm{OC}}\right)$, short-circuit current $\left(J_{\mathrm{sC}}\right)$, fill-factor $(\mathrm{FF})$ and power conversion efficiency (PCE) are shown in plots (a-d), respectively. Devices were measured under 1-sun illumination with a $0.16 \mathrm{~cm}^{2}$ aperture mask. 
for by both PL (Figs 4 and 5) and intensity-modulated photovoltage spectroscopy (IMVS) (Fig. S14) measurements. Likewise, the higher average short-circuit current densities $\left(J_{\text {sC }}\right)$ observed in the S, M and L solar cells (Fig. 6b) can be accounted for by UV-vis spectroscopy and incident photon-to-current efficiency (IPCE) measurements (Fig. S15). The implication of the present study on the efficiency of perovskite solar cells is that extending grain sizes beyond several micrometers may not have a significant bearing on solar cell efficiencies, as internal quantum efficiencies are already close to unity [45]. In this regard, efforts to improve perovskite solar cell efficiencies are best directed towards producing high-crystallinity grains of a few micrometers in diameter rather than attempting to develop millimeter-scale grains.

\section{CONCLUSION}

The present study illustrates that the nucleation and growth kinetics, which are fundamental to establishing the film morphology, can be controlled by modifying the conditions under which the solution-processed hybrid perovskite layer is formed. Through the manipulation of the solution concentration and degree of supersaturation, it is possible to vary the spacing between nucleation sites from a few hundred nanometers to a few hundred microns. Crystalline $\mathrm{CH}_{3} \mathrm{NH}_{3} \mathrm{PbI}_{3}$ regions in excess of $100 \mu \mathrm{m}$ in diameter were produced by exercising this control over the perovskite growth conditions. These crystalline regions were found to comprise multiple well-oriented grains and could not be described as single crystals. Despite the relatively slow growth rates under which these crystals form, the perovskite films featuring large grain-clusters exhibited comparatively poor optoelectronic characteristics, consistent with previous descriptions of functional materials formed through oriented-attachment. This work reveals the likely involvement of non-classical mechanisms in the growth of solution-processed perovskite thin-films, and provides a qualitative model as a framework for the design of future perovskite morphologies.

Received 16 March 2017; accepted 2 May 2017; published online 21 June 2017

1 Mitzi DB, Wang S, Feild CA, et al. Conducting layered organicinorganic halides containing-oriented perovskite sheets. Science, 1995, 267: 1473-1476

2 Mitzi DB, Chondroudis K, Kagan CR. Organic-inorganic electronics. IBM J Res Dev, 2001, 45: 29-45

3 Kojima A, Teshima K, Shirai Y, et al. Organometal halide perovskites as visible-light sensitizers for photovoltaic cells. J Am Chem Soc, 2009, 131: 6050-6051
4 Kazmerski L, Zweibel K. Best research-cell efficiencies. National Renewable Energy Laboratory (NREL) http://www.nrel.gov/ncpv/images/efficiency_chart.jpg (2014)

5 Saparov B, Mitzi DB. Organic-inorganic perovskites: structural versatility for functional materials design. Chem Rev, 2016, 116: 4558-4596

6 Wang N, Cheng L, Ge R, et al. Perovskite light-emitting diodes based on solution-processed self-organized multiple quantum wells. Nat Photon, 2016, 10: 699-704

7 Xue M, Zhou H, Xu Y, et al. High-performance ultraviolet-visible tunable perovskite photodetector based on solar cell structure. Sci China Mater, 2017, 60: 407-414

8 Berhe TA, Su WN, Chen $\mathrm{CH}$, et al. Organometal halide perovskite solar cells: degradation and stability. Energ Environ Sci, 2016, 9: 323-356

9 Niu G, Li W, Li J, et al. Progress of interface engineering in perovskite solar cells. Sci China Mater, 2016, 59: 728-742

10 Wei J, Shi C, Zhao Y, et al. Potentials and challenges towards application of perovskite solar cells. Sci China Mater, 2016, 59: 769-778

11 Ye M, Hong X, Zhang F, et al. Recent advancements in perovskite solar cells: flexibility, stability and large scale. J Mater Chem A, 2016, 4: 6755-6771

12 Burschka J, Pellet N, Moon SJ, et al. Sequential deposition as a route to high-performance perovskite-sensitized solar cells. Nature, 2013, 499: 316-319

13 Chen $\mathrm{Q}$, Zhou H, Hong Z, et al. Planar heterojunction perovskite solar cells via vapor-assisted solution process. J Am Chem Soc, 2014, 136: 622-625

14 Jeon NJ, Noh JH, Kim YC, et al. Solvent engineering for highperformance inorganic-organic hybrid perovskite solar cells. Nat Mater, 2014, 13: 897-903

15 Xiao M, Huang F, Huang W, et al. A fast deposition-crystallization procedure for highly efficient lead iodide perovskite thin-film solar cells. Angew Chem Int Ed, 2014, 53: 9898-9903

16 Huang F, Dkhissi Y, Huang W, et al. Gas-assisted preparation of lead iodide perovskite films consisting of a monolayer of single crystalline grains for high efficiency planar solar cells. Nano Energ, 2014, 10: 10-18

17 Zhou Y, Yang M, Wu W, et al. Room-temperature crystallization of hybrid-perovskite thin films via solvent-solvent extraction for high-performance solar cells. J Mater Chem A, 2015, 3: 8178-8184

18 Nie W, Tsai H, Asadpour R, et al. High-efficiency solution-processed perovskite solar cells with millimeter-scale grains. Science, 2015, 347: 522-525

19 Li X, Bi D, Yi C, et al. A vacuum flash-assisted solution process for high-efficiency large-area perovskite solar cells. Science, 2016, 353: 58-62

20 Pascoe AR, Meyer S, Huang W, et al. Enhancing the optoelectronic performance of perovskite solar cells via a textured $\mathrm{CH}_{3} \mathrm{NH}_{3} \mathrm{PbI}_{3}$ morphology. Adv Funct Mater, 2016, 26: 1278-1285

21 Im JH, Jang IH, Pellet $\mathrm{N}$, et al. Growth of $\mathrm{CH}_{3} \mathrm{NH}_{3} \mathrm{PbI}_{3}$ cuboids with controlled size for high-efficiency perovskite solar cells. Nat Nanotech, 2014, 9: 927-932

22 Hutter EM, Eperon GE, Stranks SD, et al. Charge carriers in planar and meso-structured organic-inorganic perovskites: mobilities, lifetimes, and concentrations of trap states. J Phys Chem Lett, 2015, 6: 3082-3090

23 Johnston MB, Herz LM. Hybrid perovskites for photovoltaics: charge-carrier recombination, diffusion, and radiative efficiencies. Acc Chem Res, 2016, 49: 146-154

24 D'Innocenzo V, Srimath Kandada AR, De Bastiani M, et al. Tuning the light emission properties by band gap engineering in hybrid 
lead halide perovskite. J Am Chem Soc, 2014, 136: 17730-17733

25 Pascoe AR, Yang M, Kopidakis N, et al. Planar versus mesoscopic perovskite microstructures: the influence of $\mathrm{CH}_{3} \mathrm{NH}_{3} \mathrm{PbI}_{3}$ morphology on charge transport and recombination dynamics. Nano Energ, 2016, 22: 439-452

26 Shi D, Adinolfi V, Comin R, et al. Low trap-state density and long carrier diffusion in organolead trihalide perovskite single crystals. Science, 2015, 347: 519-522

27 Zheng YC, Yang S, Chen X, et al. Thermal-induced volmer-weber growth behavior for planar heterojunction perovskites solar cells. Chem Mater, 2015, 27: 5116-5121

28 Pound GM, Mer VKL. Kinetics of crystalline nucleus formation in supercooled liquid tin. J Am Chem Soc, 1952, 74: 2323-2332

29 You H, Fang J. Particle-mediated nucleation and growth of solution-synthesized metal nanocrystals: a new story beyond the LaMer curve. Nano Today, 2016, 11: 145-167

30 Zhang Q, Liu SJ, Yu SH. Recent advances in oriented attachment growth and synthesis of functional materials: concept, evidence, mechanism, and future. J Mater Chem, 2009, 19: 191-207

31 Zhang J, Huang F, Lin Z. Progress of nanocrystalline growth kinetics based on oriented attachment. Nanoscale, 2010, 2: 18-34

32 Huang F, Zhang H, Banfield JF. Two-stage crystal-growth kinetics observed during hydrothermal coarsening of nanocrystalline $\mathrm{ZnS}$. Nano Lett, 2003, 3: 373-378

33 Zhang J, Lin Z, Lan Y, et al. A multistep oriented attachment kinetics: coarsening of $\mathrm{ZnS}$ nanoparticle in concentrated $\mathrm{NaOH}$. J Am Chem Soc, 2006, 128: 12981-12987

34 Tidhar Y, Edri E, Weissman H, et al. Crystallization of methyl ammonium lead halide perovskites: implications for photovoltaic applications. J Am Chem Soc, 2014, 136: 13249-13256

35 Tan KW, Moore DT, Saliba M, et al. Thermally induced structura evolution and performance of mesoporous block copolymer-directed alumina perovskite solar cells. ACS Nano, 2014, 8: 4730-4739

36 Ahn N, Son DY, Jang IH, et al. Highly reproducible perovskite solar cells with average efficiency of $18.3 \%$ and best efficiency of $19.7 \%$ fabricated via Lewis base adduct of lead(II) iodide. J Am Chem Soc, 2015, 137: 8696-8699

37 Lee JW, Kim HS, Park NG. Lewis acid-base adduct approach for high efficiency perovskite solar cells. Acc Chem Res, 2016, 49: 311-319

38 Zhang Y, Huang F, Mi Q. Preferential facet growth of methylammonium lead halide single crystals promoted by halide coordination.
Chem Lett, 2016, 45: 1030-1032

39 Fu Y, Meng F, Rowley MB, et al. Solution growth of single crystal methylammonium lead halide perovskite nanostructures for optoelectronic and photovoltaic applications. J Am Chem Soc, 2015, 137: 5810-5818

40 Yang $\mathrm{M}$, Zhang T, Schulz $\mathrm{P}$, et al. Facile fabrication of large-grain $\mathrm{CH}_{3} \mathrm{NH}_{3} \mathrm{PbI}_{3-x} \mathrm{Br}_{x}$ films for high-efficiency solar cells via $\mathrm{CH}_{3} \mathrm{NH}_{3} \mathrm{Br}$-selective ostwald ripening. Nat Commun, 2016, 7: 12305

41 Thompson CV. Structure evolution during processing of polycrystalline films. Annu Rev Mater Sci, 2000, 30: 159-190

42 de Quilettes DW, Vorpahl SM, Stranks SD, et al. Impact of microstructure on local carrier lifetime in perovskite solar cells. Science, 2015, 348: 683-686

43 Banfield JF, Welch SA, Zhang $\mathrm{H}$, et al. Aggregation-based crystal growth and microstructure development in natural iron oxyhydroxide biomineralization products. Science, 2000, 289: 751-754

44 Penn RL. Kinetics of oriented aggregation. J Phys Chem B, 2004, 108: $12707-12712$

45 Lin Q, Armin A, Nagiri RCR, et al. Electro-optics of perovskite solar cells. Nat Photon, 2014, 9: 106-112

46 Nývlt J. Kinetics of nucleation in solutions. J Cryst Growth, 1968 , 3-4: $377-383$

Acknowledgments The authors acknowledge the financial support from the Australian Renewable Energy Agency (ARENA), the Australian Centre for Advanced Photovoltaics (ACAP) and the ARC Centre of Excellence in Exciton Science. The authors also acknowledge the use of the facilities at Monash Centre for Electron Microscopy (MCEM).

Author contributions Pascoe A and Gu Q fabricated the perovskite films and conducted the experimental analysis. Rothmann $\mathrm{M}$ and $\mathrm{Li}$ W conducted the electron microscope imaging of the samples and analysis of diffraction patterns. Zhang Y performed the spatially resolved photoluminescence mapping. Scully A performed the time-resolved photoluminescence measurements. Lin X performed the X-ray diffraction measurements. Bach $U$ and Cheng YB contributed to the experimental design and analysis. All authors contributed to general discussion and manuscript preparation.

Conflict of interest The authors declare that they have no conflict of interest.

Supplementary information Supplementary data are available in the online version of the paper. 


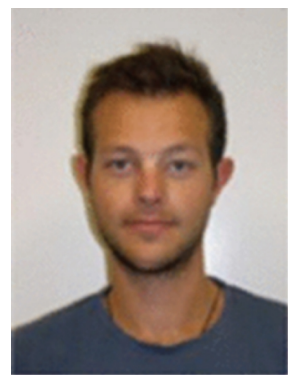

Alexander R. Pascoe received his $\mathrm{PhD}$ in materials engineering from Monash University. He was previously a research fellow at the Department of Materials Science and Engineering, Monash University. His research focused on the device physics of hybrid organic-inorganic thin film photovoltaics.

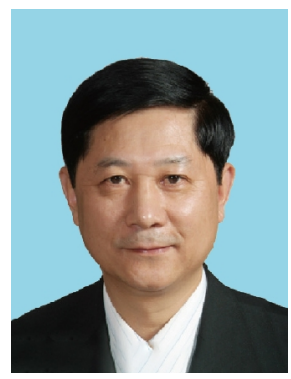

Yi-Bing Cheng is a professor in the Department of Materials Engineering at Monash University, Australia. He completed his undergraduate (1978) and Master (1983) studies at Wuhan University of Technology, China and received a PhD degree from the University of Newcastle-upon-Tyne, UK in 1989. He joined Monash University in 1991. He specializes in inorganic materials and composites. He started working on dye sensitized solar cells in 2001 and is mainly interested in solution processed solar cells. He is also a Thousand Talent Professor at Wuhan University of Technology, China.

\section{溶液法制备杂化钙铁矿薄膜过程中核化与晶粒生长动力学控制}

Alexander R. Pascoe ${ }^{1}$, 顾 钦 颖 ${ }^{1}$, Mathias U. Rothmann ${ }^{1}$, 李蔚 ${ }^{1}$, 张豫鹏 ${ }^{1}$, Andrew D. Scully ${ }^{2}$, 林雄峰 ${ }^{1}$, Leone Spiccia ${ }^{3}$, Udo Bach ${ }^{1,2}$, 程一兵 ${ }^{1,4^{*}}$

摘要 如何利用溶液法制备高质量、高效率的有机-无机杂化钙钛矿薄膜光电器件, 取决于对该体系的核化和晶体生长机理的深入研究. 尽管用很多方法可以制备出高性能的钙钛矿薄膜, 到目前为止, 还缺乏一个准确且统一的模型, 去解释钙钛矿晶体是如何从溶液中析出生 长的过程. 本文通过对晶体核化和生长动力学的详细研究, 提出了有机-无机杂化钲钛矿薄膜材料的形成机制. 通过精准控制钲钛矿晶粒生 长的条件, 异质晶核之间的距离能够在几百纳米到几百微米之间调控. 我们还发现在晶核周围直径超过 100 微米范围, 聚集着取向高度一致 的晶体团簇. 但是这些晶体团簇的尺寸大小, 与提高钲钛矿光电器件的性能并没有什么直接的对应关系; 钻钠矿材料微观结构的形成机理, 兼有经典和非经典晶体生长的特征. 因此对钲钛矿薄膜生长的深入研究, 将有助于进一步控制杂化钻钛矿薄膜的微观结构. 